\title{
VIRTUAL SCREENING OF MEDICINAL PLANT COMPOUNDS AGAINST DevR (A0R2V2) OF Mycobacterium tuberculosis USING MOLECULAR DOCKING STUDIES
}

\author{
RADHAMAHENDRAN S. ${ }^{1,2}$, MALATHI R. ${ }^{*}$, JAYARAMAN G. ${ }^{1}$ AND NISHANDHINI M. ${ }^{2}$ \\ ${ }^{1}$ Department of Genetics, University of Madras, Dr. ALM PG IBMS, Sekkizhar Campus, Chennai- 600 113, TN, India. \\ 2Department of Bioinformatics, Vels University, Chennai- 600 117, TN, India. \\ *Corresponding Author: Email- r_malathi@hotmail.com
}

Received: July 11, 2012; Accepted: July 23, 2013

\begin{abstract}
The DevR response regulator in Mycobacterium tuberculosis is believed to play a key role in bacterial dormancy adaptation during hypoxia and also shares a homologous genetic system with Mycobacterium smegmatis. Thus DevR is proposed to be an attractive target for the development of inhibitors against tuberculosis. Currently available first line anti-tuberculosis drugs have been caused several side effects in the body as well as resistance development by mycobacterium against these drugs, necessitates the considerable need for finding new drugs. Therefore, we propose a structure based computational method to find a new potential inhibitor for DevR protein. Natural sources provide numerous examples of interesting secondary metabolites with antimycobacterial activity, indicating that natural products could be a rewarding field for the discovery of new anti-TB leads. The present study is to deduce the structure of the DevR (UniprotID: A0R2V2) protein using prime module (Schrodinger) and to validate the same with Ramachandran plot. The proposed model structure is further explored for insilico docking studies with natural inhibitors obtained from published literatures which describe the effectiveness of natural anti-tuberculosis compounds. 143natural compounds were computationally analyzed using glide module for their bioactivity against DevR protein using virtual screening approach. The best ligand that exhibited high docking score with DevR was finally reported. Further, structure optimization and in vitro validation of above inhibitors will prove its efficacy as a better candidate in the drug designing pipeline.
\end{abstract}

Keywords- Tuberculosis, Virtual screening, DevR, docking, homology modelling, Mycobacterium smegmatis

Citation: RadhaMahendran S., et al. (2013) Virtual Screening of Medicinal Plant Compounds against DevR (A0R2V2) of Mycobacterium Tuberculosis using Molecular Docking Studies. International Journal of Genomics and Proteomics, ISSN: 0976-4887 \& E-ISSN: 0976-4895, Volume 4, Issue 1, pp.-72-75.

Copyright: Copyright@2013 RadhaMahendran S., et al. This is an open-access article distributed under the terms of the Creative Commons Attribution License, which permits unrestricted use, distribution and reproduction in any medium, provided the original author and source are credited.

\section{Introduction}

Tuberculosis (TB) is the leading cause of death worldwide due to an infectious disease, killing around 2 million people annually, primarily in developing countries. Over one third of the world's population is infected with TB with approximately 8 million new cases of infection every year [1]. TB incidence is also on the rise because of the correspondingly high HIV infection rates. These two diseases progress at faster rates in co-infected individuals. The emergence of multidrug-resistant strains of Mycobacterium tuberculosis (Mtb) has intensified efforts to discover novel drugs for tuberculosis (TB) treatment. Targeting the persistent state of Mtb, a condition in which Mtb is resistant to conventional drug therapies, is of particular interest. Persistent bacteria rely on metabolic pathways that are distinct from active infection Mtb as the environmental conditions of the persistent state are different (e.g., low nutrient). The emergence of extensively resistant tuberculosis has increased the failure rate and cost of treatment $[2,3]$. This has prompted further interest in the development of more effective TB treatment strategies.

Two-component systems (TCS) are majorly involved in sensing and responding to changing environments in bacteria [4]. TCS are found in bacteria, fungi, and plants $[5,6]$ and are already being exploited as new antibiotic targets $[7,8]$. These systems consist of a sensor kinase which autophosphorylates histidine, then transphosphorylates a conserved aspartate of its cognate transcriptional regulator. There is significant experimental evidence that the transcriptional regulator $\operatorname{Dev} R$ (also called $\operatorname{dos} R$ ) is a key factor in the metabolic shift-down to non-replicative persistence. Previous studies have identified DevR as a strong candidate for therapeutic intervention [9 -15]. Also it has been reported that DevR is a stationary-phase regulator required for adaptation to oxygen starvation and resistance to heat stress in M. smegmatis. [16]. Thus DevR is proposed to be an attractive target for the development of inhibitors against persistent Mycobacterial infection.

Advances in computational techniques have enabled virtual screening to have a positive impact on the discovery process. Virtual screening utilizes docking and scoring of each compound from a dataset and the technique used is based on predicting the binding modes and binding affinities of each compound in the dataset by means of docking to an X-ray crystallographic structure [17].

Virtual screening studies reported in literature stated the importance of dataset, algorithms and scoring functions. This provided us the rationale to screen plant based compounds. In this paper we report 
interaction profile of various compounds from plant sources using DevR as the target protein.

\section{Materials and Methods \\ Homology Model Development}

Homology modeling is a theoretical method that is used to predict the structure of a sequence with an accuracy that is comparable to the best results achieved experimentally. The modeled protein quality is extremely dependent on the identity between the target and template proteins. The DevR protein sequence was collected from the Uni-Prot Protein Database (UniprotID: A0R2V2).Similarity search for DevR in the Protein Data Bank (http://www.rcsb.org) was performed using the BLAST server [18]. The protein similarity search identified a very similar protein structure $3 \mathrm{C} 3 \mathrm{~W}$ which has $85 \%$ sequence identity with A0R2V2. So this structure (3C $3 \mathrm{~W})$ was used as a DevR template to generate the model. The model was generated using Prime (Schrodinger, LLC, New York, USA) [19], and then the energy was minimized using the OPLS (optimized potentials for liquid simulations) 2005force-field [20].

\section{Model Validation}

The validation of the structure model obtained from Prime was performed by inspecting the psi/phi Ramachandran Plot obtained from PROCHECK analysis [21]. In order to assess the reliability of the modeled structure of DevR, we calculated the root mean square deviation (RMSD) by superimposing it on the template structure using Pymol.

\section{Active Site Prediction}

The active site of the modeled protein was investigated using the SiteMap program [22]. This software generates information on the binding sites characteristics using novel search and analytical facilities: a SiteMap calculation begins with an initial search step that identifies or characterizes-through the use of grid points-one or more regions on the protein surface that may be suitable for binding ligands to the receptor. Contour maps are then generated, producing hydrophobic and hydrophilic maps [23]. The hydrophilic maps are further divided into donor, acceptor, and metal-binding regions. The evaluation stage, which concludes the calculation, involves assessing each site by calculating various properties: the number of site points, a measure of the size of the site; exposure/enclosure, two properties providing different measures of how available the site is to the solvent; contact, which measures how strongly the average site point-interacts with the surrounding receptor via van der Waals nonbonding interactions; donor/acceptor character, a property related to the sizes and intensities of $\mathrm{H}$-donor and $\mathrm{H}$ acceptor regions; and SiteScore, an overall property based on the previous properties, constructed and calibrated so that the average SiteScore for a promising binding site is 1.0 [23].

\section{Protein Preparation}

The protein preparation wizard of Schrodinger suite was used to prepare protein. The protein was preprocessed by deleting the substrate cofactor as well as crystallographically observed water molecules (water without $\mathrm{H}$ bonds). The Hbonds were added to the protein, atomic charges were assigned, protanation states were generated and then minimized using the OPLS 2005 force field until the average root mean square deviation for non-hydrogen atoms reaches $0.30^{\circ} \mathrm{A}$ and the resulting optimized structure was used for further studies.

\section{Ligand Preparation}

The 143 antitubercular inhibitors were retrieved from the PUBMED literature search $[24,25]$. The chemical structures of these molecules were downloaded from PubChem (http:// pubchem.ncbi.nlm.nih.gov/), few of these structures are not available in PubChem, hence we used ChemSketch version 11.01 (http:// www.acdlabs.com) to draw those structures. These ligands were prepared using LigPrep 2.3 module of Schrodinger 9.0suite which utilizes Optimized Potentials for Liquid Simulations-2005 (OPLS2005) forcefield for ligand geometry optimization. Tautomers were generated for the ligand datasets and then, neutralized. It generated the corresponding low energy 3D conformers among 32 stereoisomers per ligand [26].

\section{Structure-Based Virtual Screening}

All the ligands which were prepared using LigPrep were then subjected for docking against the molecular target DevR using Glide, version 5.5 [27] mode. Glide used a series of hierarchical filters to search for possible locations for the ligands in the active site region of the receptor. For the grid-based ligand docking, the receptor grid generation file was used. The processes of virtual screening were carried in three phases, docking simulations of varying precisions (high-throughput virtual screening (HTVS), Standard precision (SP) and extra precision (XP)) and computational intensities. Exhaustive searches were made for all the core conformations of the ligand followed by the removal of orientations having steric clashes. In the greedy based scoring phase, all the possible interactions of the ligand with the receptor were reported. Glide Extra Precision (XP) was selected in order to get a good correlation between good poses and good scores. After removing the duplicates, top 4 ligands from XP docking were considered for visual inspection.

\section{Result and Discussion}

Tuberculosis treatment with new improved drugs is a high priority to addressing the global problem of resistance to existing antitubecular drugs. DevR is a key regulator of the hypoxia-induced dormancy response in M.tb and have been tipped to be promising target for the development of new antitubercular drugs. The homology model of DevR(A0R2V2) was generated using two component transcriptional regulatory protein (PDB $3 \mathrm{C} 3 \mathrm{~W}$ ) as template. The sequence alignment of the templates to the target sequence [Fig-1] showed that the overall homology of the target sequence was greater for $3 \mathrm{C} 3 \mathrm{~W}$ by about $85 \%$. There were appreciable numbers of structurally conserved regions.

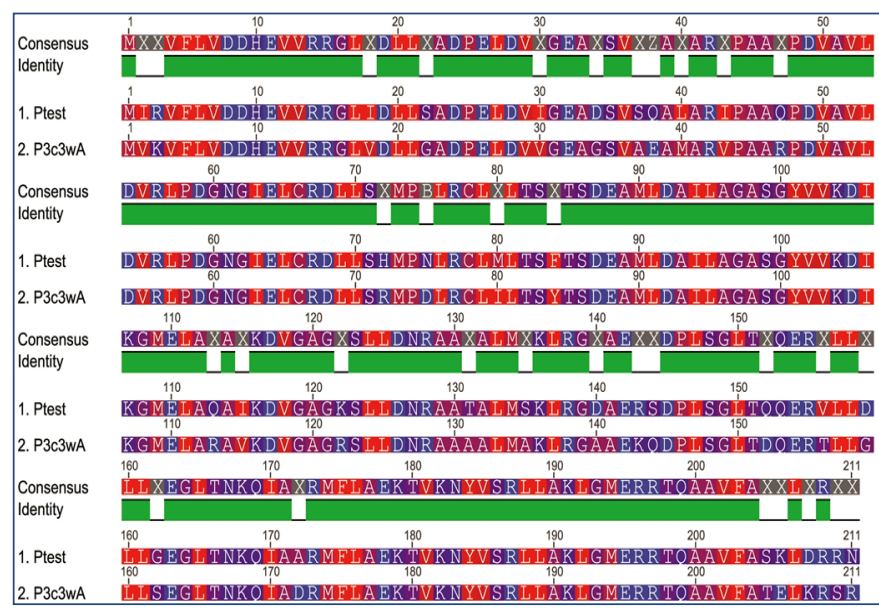

Fig. 1- Sequence alignment of AOR2V2 and 3C3W 
The homology model [Fig-2] resulted in a structure with RMSD of $<1.0$ for the heavy atoms and backbone. This may suggest a reasonable 3D model.

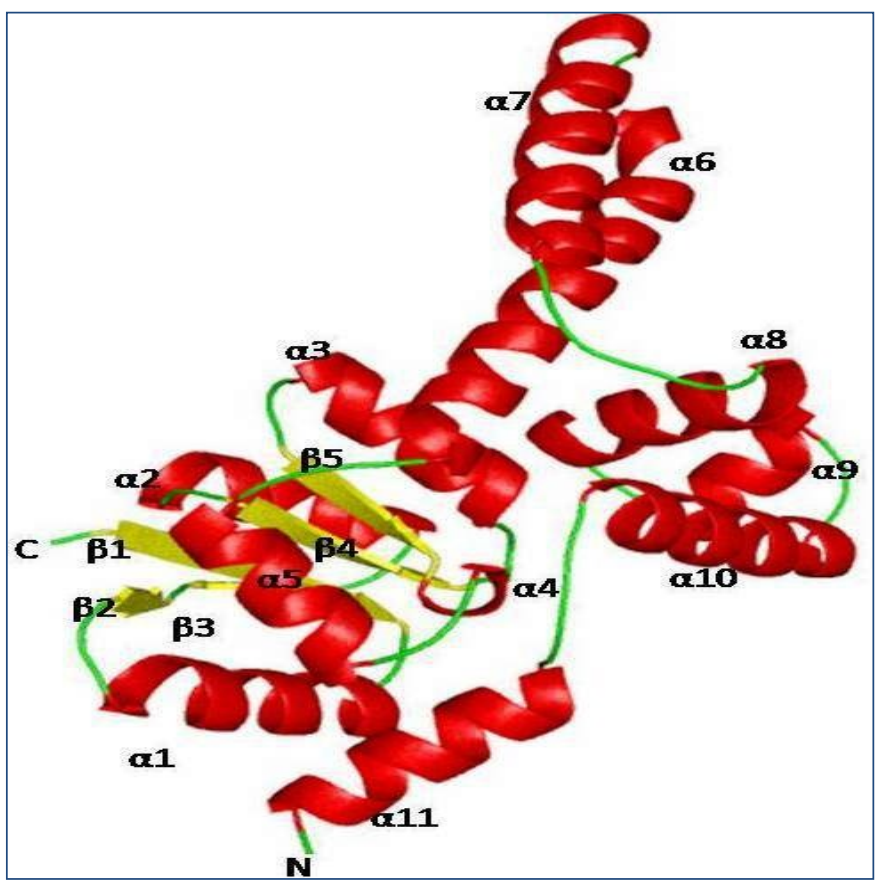

Fig. 2- Three Dimensional Structure of modelled DevR Protein

The Ramachandran plot of the DevR model from PROCHECK showed a normal distribution of points with Phi $(\Phi)$ angles mostly restricted to negative values and Psi $(\Psi)$ values clustered in a few distinct regions. It also showed that the residues in most favored region was $97.9 \%$ and residues in allowed region was $2.1 \%$ [Fig-3].

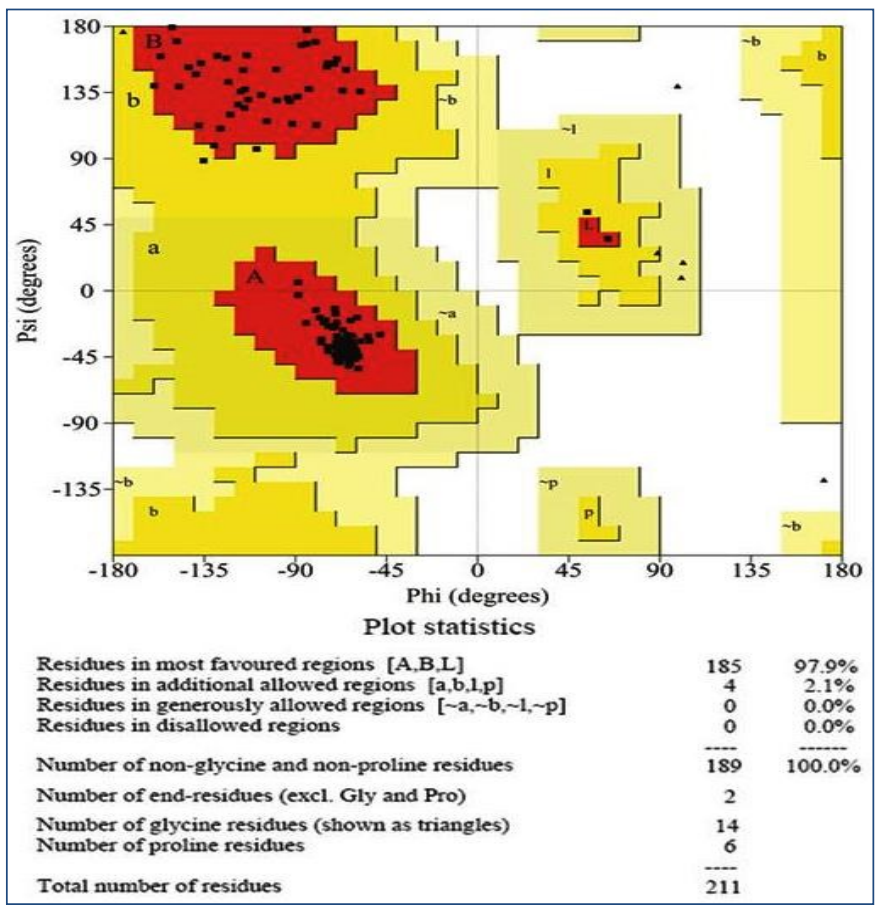

Fig. 3- Ramachandran Plot for the modelled DevR. The plot is organized as follows: Glycine, proline and all other residues are plotted as triangles, squares, and circles respectively. The red, yellow and white regions represent the favoured, allowed and the disallowed regions respectively.
The calculated root mean square deviation between the target and the template structure was found to be $0.174 \AA$ [Fig-4].

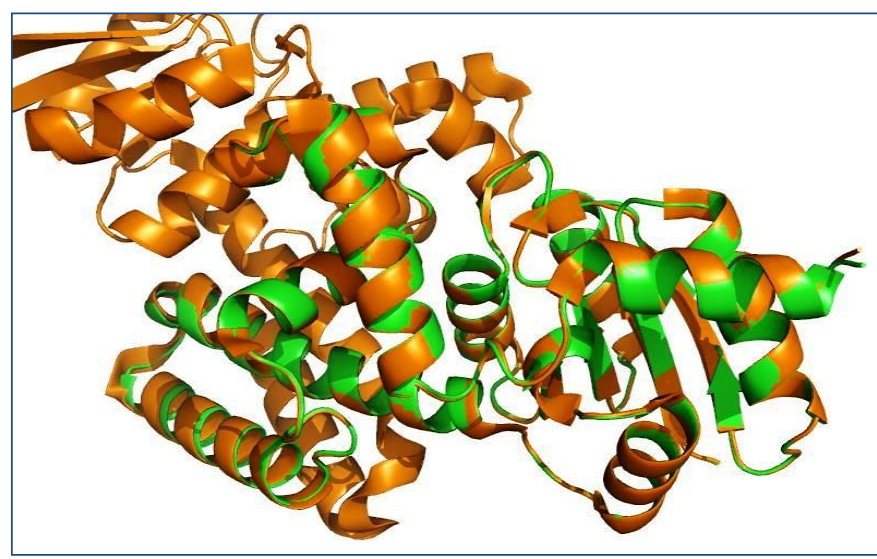

Fig. 4- Superimposition of modelled DevR (target) on $3 c 3 w$ (template). Green represents the target and yellow represents the template.

The binding site of the DevR model is made up of a narrow channel that leads to a deep cavity lined by the amino acid residues that are of great importance to the enzymes catalytic machinery. In silico predicted active sites for target protein were represented in [Fig5] (Arg56, Leu57, Pro58, Asp59, Gly60, Asn61, Leu161, Gly164, Leu165, Thr166, Asn167, Lys168, Ile170, Glu178, Lys182 and Val.185).

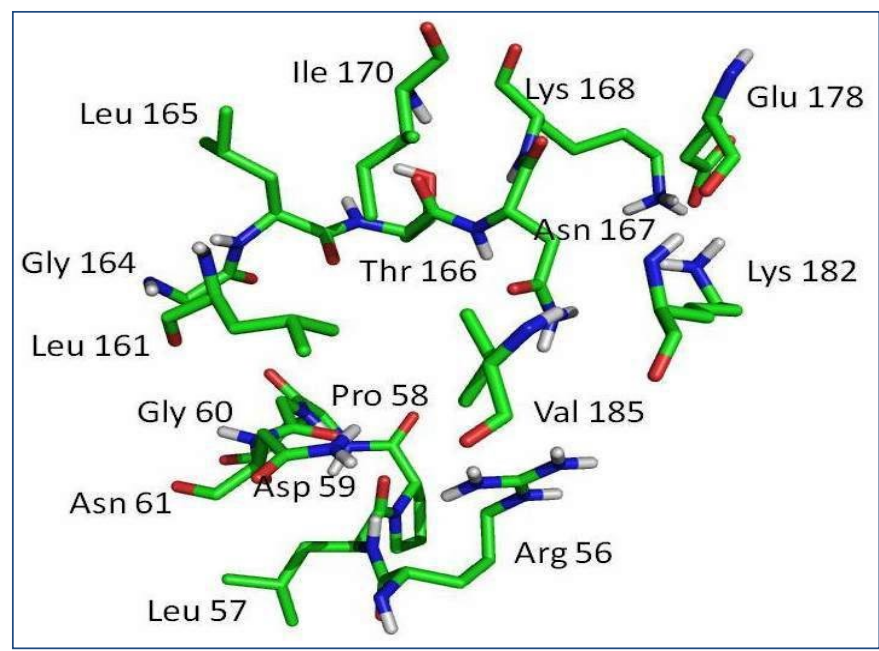

Fig. 5- Predicted binding site of target protein DevR

To gain insights into the structural basis for its activity, natural inhibitors were docked into the active site of the target protein. A correlation was calculated by Glide score and other docking parameters. For theoretical prediction of analogues, mainly three parameters were taken for consideration, these included G-score, Glide energy and hydrogen bonds. On the basis of these parameters the binding affinity of ligands toward the receptor are discussed here. From a total of 143 compounds, best four compounds were selected based on docking results. Higher negative value of G-score indicated enhanced binding affinity of the ligand with the receptor. The preferred ligands dissectol A, Goniotriol, Mangiferin and Sclareol had a Gscore value of $-8.02456,-7.426,-6.713$ and -6.559 , respectively. The docking results are revealed in [Table-1]. On analysing the docking results, all the four compounds had more than three $\mathrm{H}$ bond interactions with the receptor indicating that if the $\mathrm{H}$-bond 
interaction was more, the binding affinity of the ligand was higher. The ligand (dissectol A) had seven hydrogen bond interactions with target protein via residues Gly 60, Asn 61, Lys 182, Glu 178and Asn167. The compounds which showed very high scores and its interaction through hydrogen bonds with DevR are shown in [Fig-6]. This allows us to conclude that the compounds which have high binding score, high binding energy and have more hydrogen bonds are best inhibitors of DevR.

Table 1- Docking results of best four compounds

\begin{tabular}{|c|c|c|c|c|c|}
\hline Source & Compound & Receptor atom & Ligand atom & Distance & G score \\
\hline \multirow{7}{*}{$\begin{array}{l}\text { Incarvillea } \\
\text { dissectifoliola }\end{array}$} & \multirow[t]{7}{*}{ dissectol A } & Gly $60(0)$ & $\mathrm{H}$ & 2.83 & \multirow{7}{*}{-8.02456} \\
\hline & & Asn 61(NH) & 0 & 2.18 & \\
\hline & & Lys $182(\mathrm{NH})$ & 0 & 2.84 & \\
\hline & & Glu 178(0) & $\mathrm{H}$ & 2.82 & \\
\hline & & Asn167(0) & $\mathrm{H}$ & 2.75 & \\
\hline & & Asn 167(NH) & 0 & 3.17 & \\
\hline & & Asn 167(NH) & 0 & 2.28 & \\
\hline \multirow{5}{*}{$\begin{array}{l}\text { Goniothalamus } \\
\text { laoticus }\end{array}$} & \multirow[t]{5}{*}{ Goniotriol } & Gly $60(0)$ & $\mathrm{H}$ & 2.86 & \multirow{5}{*}{-7.426} \\
\hline & & Asn 61(NH) & 0 & 2.31 & \\
\hline & & $\operatorname{Arg} 56(\mathrm{NH})$ & 0 & 2.8 & \\
\hline & & Leu 57 (0) & 0 & 1.93 & \\
\hline & & Asn 167(NH) & 0 & 2.01 & \\
\hline \multirow{4}{*}{$\begin{array}{l}\text { Canscora } \\
\text { decussata } \\
\text { Schult. }\end{array}$} & \multirow[t]{4}{*}{ Mangiferin } & Gly $164(0)$ & $\mathrm{H}$ & 2.46 & \multirow{4}{*}{-6.713} \\
\hline & & Asn $167(\mathrm{NH})$ & 0 & 3.17 & \\
\hline & & Lys $182(\mathrm{NH})$ & 0 & 3.01 & \\
\hline & & Lys $168(\mathrm{NH})$ & 0 & 3.47 & \\
\hline \multirow{4}{*}{$\begin{array}{l}\text { Juniperus } \\
\text { excelsa }\end{array}$} & Sclareol & Gly 60(0) & $\mathrm{H}$ & 1.9 & \multirow{4}{*}{-6.559} \\
\hline & & Gly 60 (0) & $\mathrm{H}$ & 2.14 & \\
\hline & & Leu $57(0)$ & $\mathrm{H}$ & 2.18 & \\
\hline & & Asn $61(\mathrm{NH})$ & 0 & 2.87 & \\
\hline
\end{tabular}

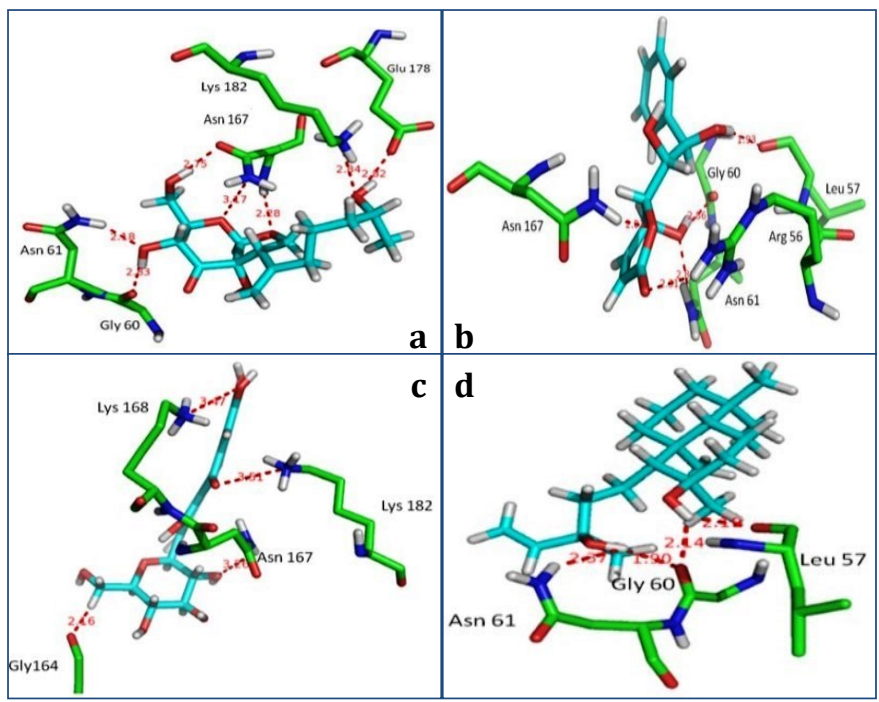

Fig. 6- $\mathrm{H}$-bond interaction between target DevR and the compounds (a) dissectol A, (b) Goniotriol, (c) Mangiferin and (d) Sclareol.

\section{Conclusion}

Screening methods are routinely and extensively used to reduce cost and time of drug discovery. It has been clearly demonstrated that the approach utilized in this study is successful in finding novel anti-tuberculosis inhibitors from plants. Compound dissectol $A$, in particular, from Incarvillea dissectifoliola showed high binding affinity against DevR.

\section{References}

[1] Dye C., Scheele S., Dolin P., Pathania V., Raviglione M.C. (1999) Journal of the American Medical Association, 282(7), 677-686.

[2] Glynn J.R., Whiteley J., Bifani P.J., Kremer K., Soolingen D.V. (2002) Emerging Infectious Diseases, 8, 843-849.

[3] Reece S.T. and Kaufmann S.H.E. (2008) International Journal of Medical Microbiology, 298, 143-150.

[4] Dasgupta N., Kapur V., Singh K.K., Das T.K., Sachdeva S., Jyothisri K. and Tyagi J.S. (2000) Tuber Lung Dis., 80(3), 141159.

[5] Stock A.M., Robinson V.L., Goudreau P.N. (2000) Annu. Rev. Biochem., 69, 183-215.

[6] Parkinson J.S., Kofoid E.C. (1992) Annu. Rev. Genet., 26, 71112.

[7] Barrett J.F. and Hoch J.A. (1998) Antimicrob Agents Chemother, 42(7), 1529-1536.

[8] Macielag M.J. and Goldschmidt (2000) Expert Opin. Investig. Drugs, 9, 2351-2369.

[9] Hasan S., Daugelat S., Rao P.S. and Schreiber M. (2006) PLoS Comput. Biol., 2, e61.

[10]Saini D.K., Malhotra V., Dey D., Pant N., Das T.K., Tyagi J.S. (2004) Microbiology, 150, 865-875.

[11]Saini D.K. and Tyagi J.S. (2005) J. Biomol. Screen, 10, $215-$ 224.

[12]Zhang Y. (2005) Annu. Rev. Pharmacol. Toxicol., 45, 529-564.

[13]Wisedchaisri G., Wu M., Rice A.E., Roberts D.M., Sherman D.R., Hol W.G. (2005) J. Mol. Biol., 354, 630-641.

[14]Zhang Y. (2004) Front Biosci., 9, 1136-1156.

[15]Zhang Y., Post-Martens K., Denkin S. (2006) Drug Discovery Today, 11, 21-27.

[16]O'Toole R., Smeulders M.J., Blokpoel M.C., Kay E.J., Lougheed K., Williams H.D. (2003) J. Bacteriol., 185(5), 1543-54.

[17]Jalaie M., Shanmugasundaram V. (2006) Mini Rev. Med. Chem., 6, 1159-1167.

[18]Altschul S.F., Madden T.L., Schaffer A.A., Zhang J., Zhang Z., Miller W., Lipman D.J. (1997) Nucleic Acids Res., 25, 33893402.

[19]Schrödinger L.L.C. (2009) Prime 2.1., New York.

[20]Jorgensen W.L., Maxwell D.S., Tirado-Rives J. (1996) J. Am. Chem. Soc., 118, 11225-11236.

[21]Laskowski R.A., MacArthur M.W., Moss D.S. and Thornton J.M. (1993) J. Appl. Crystallogr., 26, 283-291.

[22]Jørgensen A.M. and Topiol S. (2008) Chem. Biol. Drug Des., 72, 265-272.

[23]Lauria A., Ippolito M. and Almerico A.M. (2009) J. Mol. Graph Model, 27, 712-722.

[24]Gautam R., Saklani A., Jachak S.M. (2007) Journal of Ethnopharmacology, 110, 200-234.

[25]Copp B.R. and Norrie Pearce A. (2006) Nat. Prod. Rep., 24, 278-297.

[26]Schrödinger L.L.C. (2009) LigPrep 2.3, New York.

[27]Schrödinger L.L.C. (2008) Glide 5.0, New York. 